International Journal of Pure and Applied Mathematics

Volume 93 No. $6 \quad 2014,807-812$

ISSN: 1311-8080 (printed version); ISSN: 1314-3395 (on-line version)

url: http://www.ijpam.eu

doi: http://dx.doi.org/10.12732/ijpam.v93i6.7

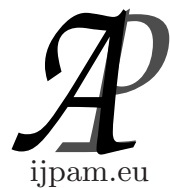

\title{
ON THE UNIQUENESS FOR THE SYMMETRIC TENSOR RANK OF TRIVARIATE POLYNOMIALS; A LOCAL UNIQUENESS FOR MULTIVARIATE POLYNOMIALS
}

\author{
E. Ballico \\ Department of Mathematics \\ University of Trento \\ 38123 Povo (Trento) - Via Sommarive, 14, ITALY
}

\begin{abstract}
For all integers $m \geq 2$ and $d \geq 3$ and $x>0$. Let $\nu_{d}: \mathbb{P}^{m} \rightarrow \mathbb{P}^{N}$, $N:=\left(\begin{array}{c}m+d \\ m\end{array}\right)-1$, be the Veronese embedding. We discuss the uniqueness (only for trivariate polynomials) and the local uniqueness of a decomposition of a polynomial into powers of linear forms in the following sense. Take $P \in \mathbb{P}^{N}$. Let $S(m, x, d, P)$ be the set of all $S \subset \mathbb{P}^{m}$ such that $\sharp(S)=x, P \in\left\langle\nu_{d}(S)\right\rangle$ (where \langle\rangle is the linear span), and $P \notin\left\langle\nu_{d}\left(S^{\prime}\right)\right\rangle$ for any $S^{\prime} \subsetneq S$. We prove that $S(m, x, d, P)=\{S\}$ (resp. $S$ is a isolated point of $S(m, x, d, P)$ ) if $m=2, x<$ $\left(d^{2}+3 d\right) / 8$ and $S$ has the general uniform position (resp. $\sharp(S) \leq\left(\begin{array}{c}m+\lfloor(d-1) / 2\rfloor \\ m\end{array}\right)$ and $S$ has general postulation). We do the same for zero-dimensional schemes (scheme rank or cactus rank).
\end{abstract}

AMS Subject Classification: 14N05

Key Words: symmetric tensor rank, trivariate polynomial, zero-dimensional scheme, multivariate polynomial

\section{Introduction}

This note is in part a continuation of [1] and we do not repeat the proofs con-

Received: March 1, 2014

(c) 2014 Academic Publications, Ltd. url: www.acadpubl.eu 
tained therein. The problem considered in [1] and in this note may be summarized as the search for explicit criteria to show the uniqueness of a solution (in this case a finite set of $\mathbb{P}^{2}$ evincing the symmetric tensor rank of a trivariate homogeneous polynomial). We recall the following terminology. Fix integers $d \geq 3$ and $m \geq 1$. Let $K$ be an algebraically closed field with either characteristic zero or characteristic $>d$. Let $K\left[x_{0}, \ldots, x_{m}\right]_{d}$ be the vector space of all homogeneous polynomials of degree $d$. For each $f \in K\left[x_{0}, \ldots, x_{m}\right]_{d} \backslash\{0\}$ the rank $r_{m, d}(f)$ is the minimal integer $t$ such that $f=\sum_{i=1}^{t} \ell_{i}^{t}$ for some $\ell_{i} \in K\left[x_{0}, \ldots, x_{m}\right]_{1}$. This notion may be translated in the following way. Let $\nu_{d}: \mathbb{P}^{m} \rightarrow \mathbb{P}^{N}$, $N:=\left(\begin{array}{c}m+d \\ m\end{array}\right)-1$, be the the order $d$ Veronese embedding of $\mathbb{P}^{m}$, i.e. the embedding of $\mathbb{P}^{m}$ induced by the complete linear system associated to $K\left[x_{0}, \ldots, x_{m}\right]_{d}$. Each $P \in \mathbb{P}^{N}$ corresponds to a polynomial $f \in K\left[x_{0}, \ldots, x_{m}\right]_{d} \backslash\{0\}$, which is unique, up to a multiplicative constant, and we set $r_{m, d}(P):=r_{m, d}(f)$. The integer $r_{m, d}(P)$ is called the rank (or the symmetric tensor rank) of $P$. The integer $r_{m, d}(P)$ is the minimal cardinality of a set $S \subset \mathbb{P}^{m}$ such that $P \in\left\langle\nu_{d}(S)\right\rangle$, where \langle\rangle denote the linear span. Using the Veronese embedding it is possible to introduce another invariant of $P$, the minimal degree $z_{m, d}(P)$ of a zerodimensional scheme $Z \subset \mathbb{P}^{m}$ such that $P \in\left\langle\nu_{d}(Z)\right\rangle$. Nowadays the integer $z_{m, d}(P)$ is often called the cactus rank of $P$ (or of any homogeneous polynomial) ([5]); it was introduced in [8], Definition 5.1, p. 135, Definition 5.66, p. 198, with the name scheme length. We say that $Z$ evinces the cactus rank (resp. the rank) of $P$ if $P \in \nu_{d}(Z)$ and $\operatorname{deg}(Z)=z_{m, d}(P)$ (resp. $Z$ is reduced and $\left.\sharp(Z)=r_{m, d}(P)\right)$. As in [1] we are only able to handle the case $m=2$ (see Theorem 2 and Remark 1). The local uniqueness problem is easier and we give a local uniqueness criterion in the multivariate case. To state it we need to introduced some jargon. Fix integers $x>0$ and $d>0$. Set $U(m, x):=$ $\left\{S \subset \mathbb{P}^{m}: \sharp(S): x\right\}$ and $U_{d}(m, x):=\left\{S \in U(m, x): \operatorname{dim}\left(\left\langle\nu_{d}(S)\right\rangle\right)=x-1\right\}$. Let $Z(m, x)$ be the set of all zero-dimensional schemes $Z \subset \mathbb{P}^{m}$ such that $\operatorname{deg}(Z)=x$. Set $Z_{d}(m, x):=\left\{S \in Z(m, x): \operatorname{dim}\left(\left\langle\nu_{d}(S)\right)=x-1\right\}\right.$. Fix $P \in \mathbb{P}^{N}, . N:=\left(\begin{array}{c}m+d \\ m\end{array}\right)-1$. Set $Z^{\prime}(m, x, d, P):=\left\{S \in Z(m, x): P \in\left\langle\nu_{d}(S)\right\rangle\right.$, $Z^{\prime \prime}(m, x, d, P):=Z^{\prime}(m, x, d, P) \cap Z_{d}(m, x), S^{\prime}(m, x, d, P):=\{S \in U(m, x):$ $P \in\left\langle\nu_{d}(S)\right\rangle, S^{\prime \prime}(m, x, d, P):=S^{\prime}(m, x, d, P) \cap Z_{d}(m, x)$. Let $Z(m, x, d, P)$ (resp. $S(m, x, d, P))$ be the set of all $S \in Z^{\prime}(m, x, d, P)$ (resp. $\left.S \in Z^{\prime}(m, x, d, P)\right)$ such that $P \notin\left\langle\nu_{d}\left(S^{\prime}\right)\right\rangle$ for any $S^{\prime} \subsetneq S$. Notice that $Z(m, x, d, P) \subseteq Z^{\prime \prime}(m, x, d, P)$ and $S(m, x, d, P) \subseteq S^{\prime \prime}(m, x, d, P)$. The schemes $U(m, x)$ and $Z(m, x)$ have a Zariski topology and hence each of their subsets inherits a topology (the induced topology of the Zariski topology of $Z(m, x)$ ). Fix $S \in Z^{\prime}(m, x, d, P)$ (resp. $\left.S \in S^{\prime}(m, x, d, P)\right)$. We say that $Z^{\prime}(m, x, d, P)$ (resp. $\left.S^{\prime}(m, x, d, P)\right)$ has local uniqueness at $S$ if $S$ is an isolated point of $Z^{\prime}(m, x, d, P)\left(\operatorname{resp} . S^{\prime}(m, x, d, P)\right)$ 
with respect to the topology just described. The same definition applies to $Z^{\prime \prime}(m, x, d, P), S^{\prime \prime}(m, x, d, P), Z(m, x, d, P)$, and $S(m, x, d, P)$ (see Remarks 2 and 3 for a collection of elementary properties). Fix $Z \in Z(m, x)$. We say that $Z$ has general Hilbert function or general postulation if for each $t \in \mathbb{N}$ either $h^{1}\left(\mathcal{I}_{Z}(t)\right)=0$ or $h^{1}\left(\mathcal{I}_{Z}(t)\right)=0$. Let $v_{x}$ be the maximal $t \in \mathbb{N}$ such that $\left(\begin{array}{c}m+t \\ m\end{array}\right) \leq x$. The scheme $Z \in Z(m, x)$ has general Hilbert function if and only if $h^{0}\left(\mathcal{I}_{Z}(t)\right)=0$ for all $t \leq v_{x}$ and $h^{1}\left(\mathcal{I}_{Z}(t)\right)=0$ for all $t>v_{x}$. We say that $Z$ has general uniform position if every $Z^{\prime} \subseteq Z$ has general postulation. We prove the following results.

Theorem 1. Fix integers $m \geq 2$ and $d \geq 3$. Fix an integer $x \leq$ $\left(\begin{array}{c}m+\lfloor(d-1) / 2\rfloor \\ m\end{array}\right)$ and assume the existence of $Z \in Z(m, x, d, P)$ with general postulation. Then $Z$ is isolated in $Z(m, x, d, P), Z^{\prime}(m, x, d, P)$, and $Z^{\prime \prime}(m, x, d, P)$. If $Z \in S(m, x, d, P)$, then it is isolated in $S(m, x, d, P), S^{\prime}(m, x, d, P)$, and $S^{\prime \prime}(m, x, d, P)$.

Theorem 2. Fix an integer $d \geq 3$ and let $S \subset \mathbb{P}^{2}$ be a finite set with general uniform position and with $k:=\sharp(S)<\left(d^{2}+2 d\right) / 8$. Fix any $P \in\left\langle\nu_{d}(S)\right\rangle$ such that $P \notin\left\langle S^{\prime}\right\rangle$ for any $S^{\prime} \subset S$ with $\sharp\left(S^{\prime}\right)=k-1$. Then $S$ is the only zerodimensional scheme $Z \subset \mathbb{P}^{2}$ such that $P \in\left\langle\nu_{d}(Z)\right\rangle$ and $\operatorname{deg}(Z) \leq k$.

Remark 1. Look at the statement of Theorem 2. If we assume $\operatorname{deg}(Z)=$ $k$, then this is the union of [1], Theorem 1.4 and Remark 2.5, except that a key assumption of [1] is almost remove. In [1] it was assumed that $r_{2, d}(P)=k$. In the statement of Theorem 2 we only assume that $P \notin\left\langle S^{\prime}\right\rangle$ for any $S^{\prime} \subset S$ with $\sharp\left(S^{\prime}\right)=x-1$. Hence we get for free that all points of $\left\langle\nu_{d}(S)\right\rangle$ have rank $x$, except the points of $x$ hyperplanes for which obviously the rank is at most $x-1$. Fix any $P\langle S\rangle$ and let $S_{1} \subseteq S$ be a minimal subset of $S$ such that $P \in\left\langle S_{1}\right\rangle$. Since every subset of $S$ is in uniform position, we may apply Theorem 2 to $S_{1}$ and get that $P$ has rank $\sharp\left(S_{1}\right)$ and that $S_{1}$ is the unique zero-dimensional scheme $A \subset \mathbb{P}^{2}$ such that $P \in\left\langle\nu_{d}(A)\right\rangle$ and $\operatorname{deg}(A) \leq \sharp\left(S_{1}\right)$, i.e. $P$ has both rank and scheme-rank equal to $\sharp\left(S_{1}\right)$ and $S_{1}$ is the only subscheme of $\mathbb{P}^{2}$ evincing the scheme-rank of $P$.

\section{Proofs and related results}

Outline of the proof of Theorem 2. Decreasing if necessary $Z$ we may assume that $P \notin\left\langle\nu_{d}\left(Z^{\prime}\right)\right\rangle$ for any $Z^{\prime} \subsetneq Z$. For any zero-dimensional scheme $U \subset \mathbb{P}^{2}$ let $h_{U}$ denotes the Hilbert function of $U$ and $D h_{U}$ its first difference. Set $W:=S \cup Z$ and $w:=\operatorname{deg}(W)$. By assumption we have $\operatorname{deg}(W) \leq 2 k$. Let 
$u$ be the only integer such that $\left(u^{2}+3 u+2\right) / 2 \leq k<\left((u+1)^{2}+3(u+1)+2\right) / 2$. Since $S$ has general uniform position we have $h^{0}\left(\mathcal{I}_{S}(u)\right)=0$ and for each $E \subseteq A$ and each integer $t \leq u$ we have $h^{0}\left(\mathcal{I}_{E}(t)\right)=\max \left\{0,\left(\begin{array}{c}t+2 \\ 2\end{array}\right)-\sharp(E)\right\}$. As in [1], Claims 2.1, 2.2, 2.3, there is a positive integer $j \leq d$ such that $D h_{W}(j)=D h_{W}(j+1)>0$ and the minimal, $m$, of such integers $j$ satisfies $m \leq u$. By [1], Claim 2.4, (which uses in an essential way either [6] or [7], Proposition at page 112), there is a degree $m$ curve $M \subset \mathbb{P}^{2}$ such that $a:=$ $\operatorname{deg}(M \cap W) \geq(m+1) d-m^{2}+m+2$. Set $A:=M \cap W$. Let $B$ be the residual scheme of $W$ with respect to $M$, i.e. the closed subscheme of $\mathbb{P}^{2}$ with $\mathcal{I}_{W}: \mathcal{I}_{M}$ as its ideal sheaf. Set $A:=M \cap D$. We have $\operatorname{deg}(B)+\operatorname{deg}(A)=w$. By [7], page 112, we also have $D h_{A}(i)+D h_{B}(i-m)=D h_{W}(i)$ for all $i$. Since $D h_{W}(d) \leq m$, we have $D h_{A}(d)=\min \left\{m, D h_{W}(d)\right\}=D h_{W}(d)$ and hence $D h_{B}(d-m)=0$. Hence $\operatorname{Res}_{M}(S)=\operatorname{Res}_{M}(Z)$ and there is a unique $Q \in\left\langle\nu_{d}(S \cap M) \cap\left\langle\nu_{d}(Z)\right\rangle\right.$ such that $P \in\left\langle\nu_{d}(S \cap M) \cup\{Q\}\right\rangle$ ([4], Lemma 8, or [3], Lemma 8). Since $\operatorname{deg}\left(\operatorname{Res}_{M}(Z)\right)=\operatorname{deg}\left(\operatorname{Res}_{M}(S)\right.$, we have $\operatorname{deg}(Z \cap M) \leq \sharp(S \cap M)$. Since $S \cap M$ has general uniform position and $\sharp(S \cap M)<k$, we may apply the inductive assumption to $Q$ and $S \cap M$ and get a contradiction.

Remark 2. Fix $S \in U(m, x)$. Since being reduced is an open condition in flat families of zero-dimension schemes, there is a neighborhood $\Omega$ of $S$ is $Z(m, x)$ such that $\Omega \subset U(m, x)$. Hence the local uniqueness property at a reduced set $S$ is the same if we look at all schemes near $S$ or just to finite sets with cardinality $x$.

Remark 3. Fix $Z \in Z(m, x)$ and assume that $Z$ has general postulation (resp. general uniform position). By the semicontinuity theorem for cohomology there is an open neighborhood $\Omega$ of $Z$ such that $W$ has general postulation (resp. general uniform position) for all $W \in \Omega$. Hence Theorem 2 is proved if we prove the following result.

Proposition 1. Fix positive integers $m, x, y, a, b, d$ such that $a+b \leq$ $d-1, x \leq\left(\begin{array}{c}m+a \\ m\end{array}\right), y \leq\left(\begin{array}{c}m+b \\ m\end{array}\right)$. Fix $P \in \mathbb{P}^{N}$ and assume the existence of $A \in$ $Z(m, x, d, P), B \in Z(m, y, d, P)$ such that $A$ and $B$ have general postulation; assume that for all $Q \in A_{\text {red }} \cap B_{\text {red }}$ (if any) $B$ is reduced at $Q$. Then $x=y$ and $A=B$.

Proof. Assume $A \neq B$. We have $h^{1}\left(\mathcal{I}_{A \cup B}(d)\right)>0$ ([2], Lemma 1). Since $A$ has general postulation and $x \leq\left(\begin{array}{c}m+a \\ m\end{array}\right)$, we have $h^{1}\left(\mathcal{I}_{A}(a)\right)=0$. CastelnuovoMumford's lemma implies that $\mathcal{I}_{A}(a+1)$ is spanned by its global sections. Hence a general $T \in\left|\mathcal{I}_{A}(a+1)\right|$ meets no connected component of $B$ which does not intersect $A$. Since $B$ is reduced at each point of $A_{\text {red }} \cap B_{\text {red }}$, we get 
that $T \cap(A \cup B)=A$ as schemes. Hence we have an exact sequence

$$
0 \rightarrow \mathcal{I}_{B \backslash B \cap A_{\text {red }}}(d-a-1) \rightarrow \mathcal{I}_{A \cup B}(d) \rightarrow \mathcal{I}_{A, T}(d) \rightarrow 0
$$

We have $h^{1}\left(T, \mathcal{I}_{A, T}(d)\right)=h^{1}\left(\mathcal{I}_{A}(d)\right)=0$. Since $b \leq d-a-1, y \leq\left(\begin{array}{c}m+b \\ m\end{array}\right)$ and $B$ has general postulation, we have $h^{1}\left(\mathcal{I}_{B}(d-a-1)\right)=0$. Since $B \backslash B \cap A_{\text {red }} \subseteq B$, we have $h^{1}\left(\mathcal{I}_{B \backslash B \cap A_{\text {red }}}(d-a-1)\right)=0$. Hence $(1)$ gives $h^{1}\left(\mathcal{I}_{A \cup B}(d)\right)=0$, a contradiction.

\section{Acknowledgements}

The author was partially supported by MIUR and GNSAGA of INdAM (Italy).

\section{References}

[1] E. Ballico and L. Chiantini, A criterion for detecting the identifiability of symmetric tensors of size three, Differential Geom. Appl. 30 (2012), no. 3, 233-237.

[2] E. Ballico and A. Bernardi, Decomposition of homogeneous polynomials with low rank, Math. Z. 271 (2012), 1141-1149; DOI 10.1007/s00209-0110907-6.

[3] E. Ballico and A. Bernardi, A partial stratification of secant varieties of Veronese varieties via curvilinear subschemes, Sarajevo J. Math. 8 (2012), no. $1,33-52$.

[4] E. Ballico and A. Bernardi, Stratification of the fourth secant variety of Veronese variety via the symmetric rank, Adv. Pure Appl. Math. 4 (2013), no. 2, 215-250; DOI: 10.1515/apam-2013-0015

[5] A. Bernardi and K. Ranestad, The cactus rank of cubic forms, J. Symbolic. Comput. 50 (2013) 291-297. DOI: 10.1016/j.jsc.2012.08.001

[6] E. D. Davis, 0-dimensional subschemes of $\mathbf{P}^{2}$ : new application of Castelnuovo's function. Ann. Univ. Ferrara Sez. VII (N.S.) 32 (1986), 93-107 (1987).

[7] Ph. Ellia and Ch. Peskine, Groupes de points de $\mathbf{P}^{2}$ : caractère et position uniforme, in: Algebraic geometry (L'Aquila, 1988), 111-116, Lecture Notes in Math., 1417, Springer, Berlin, 1990. 
[8] A. Iarrobino and V. Kanev. Power sums, Gorenstein algebras, and determinantal loci. Lecture Notes in Mathematics, vol. 1721, Springer-Verlag, Berlin, 1999, Appendix C by Iarrobino and Steven L. Kleiman. 